\title{
Ensino-aprendizagem de programação: uma revisão sistemática da literatura
}

\author{
Teaching-learning of programming: a systematic literature review
}

\author{
Thiago Reis da Silva \\ PPgSC - Universidade Federal do \\ Rio Grande do Norte (UFRN) \\ thiagoreis@ppgsc.ufrn.br \\ Ranyer Lopes \\ PPgSC - Universidade Federal do \\ Rio Grande do Norte (UFRN) \\ ranyer@ppgsc.ufrn.br
}

\author{
Taina Jesus Medeiros \\ PPgSC - Universidade Federal do \\ Rio Grande do Norte (UFRN) \\ tainamedeiros@ppgsc.ufrn.br \\ Eduardo Aranha \\ DIMAp - Universidade Federal \\ do Rio Grande do Norte (UFRN) \\ eduardohsaranha@dimap.ufrn.br
}

\author{
Handerson Medeiros \\ PPgSC - Universidade Federal do \\ Rio Grande do Norte (UFRN) \\ handersonmedeiros@gmail.com
}

Resumo Iniciativas internacionais indicam a importância do ensino de programação desde o ensino básico, não somente para formação de novos profissionais na área de tecnologia, mas também em outras áreas, como engenharia e matemática. Ainda existem, porém diversos desafios para a implantação desta ideia. Nesta perspectiva, este artigo tem como objetivo apresentar uma revisão sistemática da literatura referente às abordagens para o ensino-aprendizagem de programação, publicados nos últimos cinco anos (2009 a 2013), nos quatro mais importantes eventos nacionais da área, o Simpósio Brasileiro de Informática na Educação, o Workshop de Informática na Escola, Workshop de Educação em Computação, e o Simpósio Brasileiro de Jogos e Entretenimento Digital, além das duas mais relevantes revistas nacionais na área, a Revista Brasileira de Informática na Educação e a Revista Novas Tecnologias na Educação. Os resultados obtidos mostram uma preferência dos pesquisadores por desenvolvimento de ferramentas para o ensino de algoritmos e suas pesquisas focam principalmente no ensino-aprendizagem de programação para o ensino superior, no contexto do ensino presencial, sendo a maioria realizada por pesquisadores localizados nas regiões Nordeste, Sul e Sudeste do Brasil. Os resultados mostram também que as publicações nesta área de pesquisa aumentaram em 2013.

Palavras-Chave: Ensino de Programação, Ensino Básico e Superior, Revisão Sistemática

\begin{abstract}
International initiatives highlight the importance of teaching programming in elementary education, not only for training new professionals in the technology area, but in other areas too, such as engineering and mathematics. However, there are still many challenges to implementing this idea. In this perspective, this article presents a systematic review of the literature related to approaches to learning and teaching programming, and that was published over the last five years (2009-2013) in four major national events in the area (the Brazilian Symposium on Informatics in Education, the Workshop on Computers in School, Workshop on Computer Education and the Brazilian Symposium on Games and Digital Entertainment) and in two major national journals in the area (the Brazilian Journal of Computing in Education Magazine and the New Technologies in Education Journal). The observed results show that researchers prefer to develop tools for teaching algorithms and their research focus mainly on teaching programming to college education in a classroom teaching context, mostly carried out by researchers located in the Northeast, South and Southeast of Brazil. The results also show that publications in this research area have increased in 2013.
\end{abstract}

Keywords: Teaching Programming, Secondary and Higher Education, Systematic Review 


\section{Introdução}

Na sociedade um elemento fundamental é a educação, que visa a criação de competências para sua atuação efetiva no mercado de trabalho e na sua formação como auto aprendiz para se adaptar à rápida mudança tecnológica e para aproveitar o conhecimento adquirido no complexo processo de tomada de decisão [17].

Os últimos anos têm sido marcados pelo desenvolvimento de novos recursos para auxiliar o processo de ensino-aprendizagem de programação. No Brasil, o aprendizado de conceitos de programação é reservado em geral para aqueles que optam por cursos de graduação na área ou cursos correlatos [18]. Esse cenário é diferente em outros países, a exemplo da Estônia, que implantou um programa para o ensino de programação para alunos com idade a partir dos sete anos [29], e os Estados Unidos [30], têm se preocupado em formar essas competências desde cedo, a partir das séries iniciais. Isto porque segundo [19], os governos desses países entendem que isso gerará impactos nas suas economias e na capacidade que seus cidadãos terão para se adaptar às mudanças. Porém, o Grupo de Licenciatura em Computação (GT-3) da Sociedade Brasileira de Computação (SBC) tem suportado a proposta de incluir conteúdos de informática no Ensino Médio [17], apesar disso ainda não ser uma realidade.

De fato, como relata o Model Curriculum for $\mathrm{K}-12$ Computer Science [30], a grande maioria das profissões do Século 21 exige uma compreensão da ciência da computação, principalmente no que tange à programação. E para conscientizar as pessoas quanto à necessidade deste conhecimento, os irmãos Ali e Hadi Partovi criaram a Code.Org ${ }^{1}$, uma Organização não Governamental (ONG) que incentiva o ensino de programação para todos, mas em especial para os estudantes do ensino básico. Através desta iniciativa, personalidades como Mark Zuckerberg (criador do Facebook), Bill Gates (fundador da Microsoft), Jack Dorsey (inventor do Twitter) e outros nomes, relatam que programar é uma habilidade extremamente importante e que está sendo cada vez mais exigida. Outras iniciativas, como, CodeHS ${ }^{2}$, Codecademy ${ }^{3}$ e Bootstrap ${ }^{4}$, também incentivam o ensino de programação.

Os resultados de estudos realizados na área, particularmente em Jogos [7][14], Robótica [16][20], Ferramentas [1][21], Metodologias [11][13] e Técnicas [15][6], indicam que os alunos se sentem muito empenhados e

\footnotetext{
${ }^{1} \mathrm{http}: / /$ code.org/

${ }^{2}$ https://codehs.com/

${ }^{3} \mathrm{http}: / /$ www.codecademy.com/pt

${ }^{4} \mathrm{http}: / /$ getbootstrap.com/
}

motivados por essa nova experiência de aprendizagem, produzindo níveis mais altos de ativação de estudante, mais emoções positivas, melhores atitudes em relação ao material de aprendizagem e maiores sucessos de aprendizagem do que os métodos convencionais de ensino. Entretanto, a aplicação efetiva e em escala do ensino de programação no ensino básico ainda requer mais pesquisa e o desenvolvimento de novas abordagens.

Para fundamentar novas pesquisas que insiram de forma efetiva o ensino de programação na educação básica, é importante o conhecimento dos trabalhos relacionados existentes e das experiências reportadas que considerem as características e desafios encontrados no cenário do ensino nacional. Por exemplo, é importante se observar quais abordagens vêm sendo propostas e para qual público alvo, bem como os resultados obtidos e limitações observadas. De fato, são várias as possibilidades de se trabalhar programação nas escolas e que precisam ser investigadas: aprendizado independente ou em sincronia com assuntos vistos em sala de aula; uso de diferentes abordagens pedagógicas; habilidade e competências que podem ser exploradas; ensino presencial ou à distância; dentre outros.

O trabalho proposto em [2] apresenta uma revisão sistemática de ensino de programação para iniciantes. Entretanto, a mesma abrange somente duas conferências nacionais, deixando de fora revistas e outras conferências relevantes. Esse estudo relacionado também não apresenta uma avaliação da qualidade dos trabalhos lidos e suas questões de pesquisa não abrangem certos aspectos cobertos por este trabalho (ensino presencial ou à distância, habilidade e competências estão sendo exploradas, entre outros).

Por essas razões, este trabalho investiga a literatura brasileira sobre a utilização de abordagens para o ensinoaprendizagem de programação como auxílio no processo educacional. Para esse fim e almejando o alcance de resultados de valor científico, optou-se pela Revisão Sistemática da Literatura (RSL). Uma RSL é uma pesquisa em profundidade de um fenômeno de interesse que produz resultados específicos e detalhados por meio da análise de conteúdo e qualidade do material pesquisado [8].

Para reportar o trabalho realizado, o resultado deste artigo encontra-se organizado da seguinte forma. $\mathrm{Na}$ Seção 2 é apresentado o método utilizado para a RSL, as questões de pesquisa, o processo de busca, os critérios de inclusão e exclusão, avaliação da qualidades, a coleta e análise dos dados e o procedimento de distribuição e análise dos artigos. Já a Seção 3 aborda os resultados gerais obtidos a partir da execução da RSL. A Seção 4 
discute detalhadamente as questões de pesquisa de acordo com os resultados encontrados e as limitações do estudo. Por fim, na Seção 5 são descritas as conclusões.

\section{Método}

Esta RSL está estruturada com base nas diretrizes originais propostas por Kitchenham et. al [8]. No caso desta revisão, objetivo é fazer uma análise do panorama atual das pesquisas e experiências práticas em relação às abordagens de ensino-aprendizagem de programação no contexto nacional entre os anos de 2009 a 2013.

\subsection{Questões de pesquisa} são:

As questões de pesquisa abordadas por este estudo

- QP1: Quais as abordagens (metodologia, ferramentas, jogo, robótica, técnica e outras) estão sendo propostas para o ensino de programação?

- QP2: Quais os níveis de escolaridade focados por estas abordagens? Ou seja, as abordagens propostas estão sendo aplicadas nas escolas e/ou em universidades?

- QP3: Quais áreas de conhecimento estão sendo trabalhadas pelas abordagens propostas? Ou seja, existe relação entre estas abordagens e o conteúdo visto em sala de aula?

- QP4: Quais abordagens pedagógicas estão sendo utilizadas nos estudos?

- QP5: Quais habilidades e competências estão sendo exploradas nos alunos?

- QP6: Quais benefícios e limitações estão sendo relatados pelo uso das abordagens propostas?

- QP7: As abordagens estão sendo destinadas à Educação Presencial e/ou à Distância?

- QP8: Quais são as instituiçõos de pesquisa envolvidas na área?

\subsection{Processo de busca}

O processo de pesquisa escolhido foi a busca manual dos anais das conferências e revistas mais relevantes desta área, publicadas entre 2009 e 2013. As revistas e conferências selecionadas são apresentadas na Tabela 1 . Os eventos e revistas foram selecionados por serem consideradas importantes bases de pesquisa nacional na área e por incluir tanto trabalhos teóricos, como práticos, além de terem sido usados como fonte para outras RSLs: [2], [3], [22], [23] e [25].

\begin{tabular}{|c|c|}
\hline Fonte & Acrônimo \\
\hline $\begin{array}{c}\text { Revista Brasileira de Informática na } \\
\text { Educação }\end{array}$ & RBIE \\
\hline Revista Novas Tecnologias na Educação & RENOTE \\
\hline Workshop de Informática na Escola & WIE \\
\hline Workshop de Educação em Computação & WEI \\
\hline $\begin{array}{c}\text { Simpósio Brasileiro de Informática na } \\
\text { Educação }\end{array}$ & SBIE \\
\hline $\begin{array}{c}\text { Simpósio Brasileiro de Jogos e Entrete- } \\
\text { nimento Digital }\end{array}$ & SBGAMES \\
\hline
\end{tabular}

Tabela 1: Revistas e conferências pesquisadas.

Uma abordagem em duas etapas foi utilizada no processo de busca. A primeira etapa envolveu a seleção de estudos baseados em seus títulos, resumos e palavraschaves. Foram considerados como estudos irrelevantes e foram removidos aqueles estudos que não continham nos títulos, resumos e palavras-chave alguma indicação de que o trabalho se se caracterizava como relevante para o estudo. No caso de dúvida sobre a relevância do artigo com base apenas em seu título, o estudo foi mantido. A segunda etapa envolveu a seleção de estudos com base em seus resumos e conclusões. É importante ressaltar que, em alguns casos, a leitura de outras partes dos artigos foi realizada para se proceder à Seleção.

\subsection{Critérios de inclusão e exclusão}

A pesquisa foi realizada através de busca manual nos anais das conferências e revistas apresentado na Tabela 1, publicados nos últimos cinco anos. Foram incluídos artigos de 1 de janeiro de 2009 a 31 de dezembro de 2013. Esta busca foi executada em duas etapas, apresentado na Subseção 2.2. Nestas duas etapas foram aplicados os critérios de inclusão e exclusão apresentados na Tabela 2. Os estudos semelhantes que apresentavam a evolução de uma mesma pesquisa e que continham os mesmos autores tiveram suas versões mais antigas removidas para evitar duplicações. Após isto, os dados extraídos dos artigos selecionados foram sintetizados para análise e apresentação dos resultados.

\begin{tabular}{|l|l|}
\hline \multicolumn{1}{|c|}{ Critérios de inclusão } & \multicolumn{1}{|c|}{ Critérios de Exclusão } \\
\hline $\begin{array}{l}\text { Artigos completos e resu- } \\
\text { mos estendidos que apre- } \\
\text { sentam abordagens de } \\
\text { ensino de programação; e }\end{array}$ & $\begin{array}{l}\text { Estudos fora dos critérios } \\
\text { de inclusão; e } \\
\text { Artigos derivados da } \\
\text { mesma pesquisa (estudos } \\
\text { duplicados). }\end{array}$ \\
$\begin{array}{l}\text { Relato de experiência, } \\
\text { estudos empíricos ou apli- } \\
\text { cação de alguma aborda- } \\
\text { gem para o ensino de } \\
\text { programação. }\end{array}$ \\
\hline
\end{tabular}

Tabela 2: Critérios de inclusão e exclusão. 


\subsection{Avaliação da qualidade}

As questões de avaliação abaixo foram aplicadas para se obter uma pontuação final para cada artigo. O procedimento de pontuação das questões de avaliação foi de $S$ $(\operatorname{sim})=1, \mathrm{P}($ parcialmente $)=0.5$ e N $($ não $)=0$, conforme sugerido em [8]. Na RSL foi utilizada quatro questões de avaliação (QA):

- QA1. Foi utilizada alguma ferramenta para auxiliar a abordagem apresentada?

- QA2. Foi utilizada alguma metodologia de desenvolvimento para dar suporte à abordagem apresentada?

- QA3. Foi realizado algum tipo avaliação experimental?

- QA4. O estudo avaliado apresenta uma comparação com outras metodologias usadas atualmente?

\subsection{Coleta e análise de dados}

Nesta fase, foram extraídos os seguinte dados sobre os artigos selecionados: título, evento, ano, autores, instituições de pesquisa, unidades federativas destas instituições, nível de escolaridade, disciplina e curso onde a pesquisa foi aplicada. Foram extraídos também, trechos dos artigos que ajudaram a responder as perguntas de pesquisa desta revisão: abordagens propostas, avaliação da qualidade, resumo do estudo, incluindo as principais questões de pesquisas e suas respostas, limitações, benefícios, habilidades e competências e sugestões para pesquisas futuras.

\subsection{Procedimentos de distribuição e análise dos artigos}

A partir da lista de publicações identificadas, os artigos foram atribuídos de forma aleatória pelo pesquisador doutor R5 para outros quatros pesquisadores, quatro alunos de pós-graduação (R1, R2, R3 e R4). Cada pesquisador avalia individualmente seus artigos e os resultados de R1, R2, R3 e R4 foram integrados por R5 na tabela de Concordância/Discordância. Por fim, R5 julga e resolve as discordâncias na tabela, numa lista final de estudos avaliados. Os pesquisadores R1, R2 e R3 analisaram 15 artigos distintos, já R4 e R5 analisaram 14 artigos cada um. Desta forma, cada artigo foi lido por apenas um revisor.

\section{Resultados}

Esta seção resume os resultados alcançados pelo estudo

\subsection{Números da pesquisa}

Como resultado de busca baseadas em títulos e resumos, de uma total de 2325 artigos, foram préselecionados 105 estudos, como mostrado na Tabela 3 . Destes, foram excluídos 32 com base nos critérios de inclusão e exclusão, resultando em 73 estudos relevantes, selecionados para a extração de dados. As referências bibliográficas destes artigos podem ser vistas no Apêndice A.

\begin{tabular}{|c|c|c|c|c|}
\hline Fonte & $\begin{array}{c}\text { Total de } \\
\text { artigos }\end{array}$ & $\begin{array}{c}\text { Artigos } \\
\text { Incluídos }\end{array}$ & $\begin{array}{c}\text { Artigos pré- } \\
\text { selecionados }\end{array}$ & $\begin{array}{c}\text { Artigos } \\
\text { incluídos } \\
(\%)\end{array}$ \\
\hline SBIE & 548 & 24 & 30 & $4.38 \%$ \\
\hline WIE & 252 & 12 & 18 & $4.76 \%$ \\
\hline WEI & 176 & 25 & 38 & $14,20 \%$ \\
\hline RBIE & 106 & 2 & 4 & $1.88 \%$ \\
\hline RENOTE & 689 & 7 & 9 & $1.02 \%$ \\
\hline SBGAMES & 554 & 3 & 6 & $0.54 \%$ \\
\hline Total & $\mathbf{2 3 2 5}$ & $\mathbf{7 3}$ & $\mathbf{1 0 5}$ & $\mathbf{2 6 . 7 8 \%}$ \\
\hline
\end{tabular}

Tabela 3: Resultado das buscas.

\subsection{Avaliação da qualidade dos estudos re- portados}

Foram avaliados os estudos usando os critérios estabelecidos na Subseção 2.4. A pontuação de cada estudo é apresentada na Tabela 4. Os resultados da análise mostram que a qualidade dos estudos teve uma média de dois pontos. Apenas três estudos (E7, E31 e E54) obtiveram pontuação inferior à média, ou seja, nota um, mas os mesmos foram mantidos na RSL. Somente dois estudos obtiveram a nota máxima, ou seja, quatro (E12 e E15).

\section{Discussões}

Nesta seção, apresentam-se as respostas às questões de pesquisa desta RSL.

\subsection{Quais as abordagens estão sendo propos- tas para o ensino de programação?}

Para fornecer um quadro geral sobre as abordagens apresentadas nos estudos, foram definidas categorias que pudessem classificar as mesmas. Como não se conhece uma classificação padrão que se encaixasse à necessidade desta revisão, foram definidas algumas categorias, tomando-se como base as categorias propostas por [2]. As abordagens propostas nos estudos foram então classificadas e brevemente descritas, conforme apresentadas na Tabela 5 . 
Nos dados apresentados, 45\% dos artigos propõem ferramentas de software para o ensino-aprendizagem de programação, $20 \%$ propõe jogos, $14 \%$ propõe a utilização de metodologias e $12 \%$ sugere a utilização da robótica. Os outros tipos de abordagens, como linguagem de programação, arquitetura pedagógica e técnicas avaliação, representam $9 \%$ dos estudos.

Os dados indicam uma preferência pelo desenvolvimento de ferramentas, para auxiliar o processo de ensinoaprendizagem de programação. Além disso, os jogos têm ganhando destaque em pesquisas recentes. Novas metodologias e robótica também estão sendo trabalhadas no ensino-aprendizagem de programação.
Nesta revisão verificaram-se estudos que trabalham com mais de uma abordagem, por exemplo, a utilização conjunta de jogos e robótica (E26 e E34) e ferramenta e jogo (E31).

Além de observar qual abordagem está sendo utilizada, é fundamental compreender o nível de avaliação (estudo de caso e/ou experimento) realizado por cada pesquisa para validar o processo de ensino de programação. Os estudos selecionados apresentam oficinas de jogos e robóticas (8), experimentos de curto e longo período nas escolas e universidades (5), estudos de caso (3), aplicação de questionários (15) e técnica de tempestade de ideias (brainstorming) (1).

\begin{tabular}{|c|c|c|c|c|c|c|c|c|c|c|c|}
\hline ESTUDOS & QA1 & QA2 & QA3 & QA4 & PONTOS & ESTUDOS & QA1 & QA2 & QA3 & QA4 & PONTOS \\
\hline E1 & $\mathrm{N}$ & $\mathrm{S}$ & $\mathrm{S}$ & $\mathrm{N}$ & 2 & E38 & $\mathrm{N}$ & $\mathrm{N}$ & $\mathrm{S}$ & $\mathrm{S}$ & 2 \\
\hline E2 & $\mathrm{N}$ & $\mathrm{N}$ & $S$ & $\mathrm{~S}$ & 2 & E39 & $\mathrm{N}$ & $\mathrm{N}$ & $\mathrm{S}$ & $\mathrm{S}$ & 2 \\
\hline E3 & $\mathrm{S}$ & $\mathrm{N}$ & $\mathrm{S}$ & $\mathrm{S}$ & 3 & E40 & $\mathrm{N}$ & $\mathrm{N}$ & $\mathrm{S}$ & $\mathrm{S}$ & 2 \\
\hline E4 & $\mathrm{N}$ & $\mathrm{N}$ & $\mathrm{S}$ & $\mathrm{S}$ & 2 & E41 & $\mathrm{S}$ & $\mathrm{N}$ & $\mathrm{S}$ & $\mathrm{S}$ & 3 \\
\hline E5 & $\mathrm{S}$ & $\mathrm{N}$ & $S$ & $\mathrm{~N}$ & 2 & E42 & S & $\mathrm{N}$ & $\mathrm{S}$ & $\mathrm{S}$ & 3 \\
\hline E6 & $\mathrm{N}$ & $\mathrm{S}$ & $\mathrm{S}$ & $\mathrm{N}$ & 2 & E43 & $\mathrm{S}$ & $\mathrm{N}$ & $\mathrm{S}$ & $\mathrm{S}$ & 3 \\
\hline E7 & $\mathrm{N}$ & $\mathrm{N}$ & $\mathrm{S}$ & $\mathrm{N}$ & 1 & E44 & $S$ & $\mathrm{~N}$ & $\mathrm{~S}$ & $\mathrm{~N}$ & 2 \\
\hline E8 & $\mathrm{S}$ & $\mathrm{N}$ & $S$ & $\mathrm{~N}$ & 2 & E45 & S & $\mathrm{N}$ & $\mathrm{P}$ & $\mathrm{P}$ & 2 \\
\hline E9 & $\mathrm{S}$ & $\mathrm{N}$ & $\mathrm{S}$ & $\mathrm{S}$ & 3 & E46 & $\mathrm{S}$ & $\mathrm{N}$ & $\mathrm{N}$ & $\mathrm{S}$ & 2 \\
\hline E10 & $\mathrm{N}$ & $\mathrm{S}$ & $\mathrm{S}$ & $\mathrm{S}$ & 3 & E47 & $\mathrm{S}$ & $\mathrm{N}$ & $\mathrm{S}$ & $\mathrm{S}$ & 3 \\
\hline E11 & $\mathrm{S}$ & $\mathrm{N}$ & $S$ & $\mathrm{~S}$ & 3 & E48 & S & $\mathrm{S}$ & $\mathrm{N}$ & $\mathrm{S}$ & 3 \\
\hline E12 & $\mathrm{S}$ & $\mathrm{S}$ & $\mathrm{S}$ & $\mathrm{S}$ & 4 & E49 & $S$ & $\mathrm{~N}$ & $\mathrm{~N}$ & $\mathrm{~S}$ & 2 \\
\hline E13 & $\mathrm{N}$ & $\mathrm{N}$ & $\mathrm{S}$ & $\mathrm{S}$ & 2 & E50 & $S$ & $\mathrm{~N}$ & $\mathrm{~S}$ & $\mathrm{~S}$ & 3 \\
\hline E14 & $\mathrm{S}$ & $\mathrm{N}$ & $\mathrm{S}$ & $\mathrm{N}$ & 2 & E51 & $\mathrm{N}$ & $\mathrm{N}$ & $\mathrm{S}$ & $\mathrm{S}$ & 2 \\
\hline E15 & $\mathrm{S}$ & $\mathrm{S}$ & $S$ & $\mathrm{~S}$ & 4 & E52 & $\mathrm{N}$ & $\mathrm{N}$ & S & $\mathrm{S}$ & 2 \\
\hline E16 & $\mathrm{N}$ & $\mathrm{N}$ & $\mathrm{S}$ & $\mathrm{S}$ & 2 & E53 & $\mathrm{S}$ & $\mathrm{N}$ & $\mathrm{S}$ & $\mathrm{S}$ & 3 \\
\hline E17 & $\mathrm{N}$ & $\mathrm{S}$ & $S$ & $\mathrm{~N}$ & 2 & E54 & $\mathrm{N}$ & $\mathrm{N}$ & $\mathrm{N}$ & $\mathrm{S}$ & 1 \\
\hline E18 & $\mathrm{S}$ & $\mathrm{S}$ & $\mathrm{N}$ & $\mathrm{S}$ & 3 & E55 & $\mathrm{S}$ & $\mathrm{N}$ & $\mathrm{S}$ & $\mathrm{S}$ & 3 \\
\hline E19 & $\mathrm{N}$ & $\mathrm{N}$ & $\mathrm{S}$ & $\mathrm{S}$ & 2 & E56 & $S$ & $\mathrm{~N}$ & $\mathrm{~N}$ & $\mathrm{~S}$ & 2 \\
\hline E20 & $\mathrm{N}$ & $\mathrm{N}$ & $S$ & $\mathrm{~S}$ & 2 & E57 & $S$ & $\mathrm{~S}$ & $\mathrm{~N}$ & $\mathrm{~N}$ & 2 \\
\hline E21 & $\mathrm{S}$ & $\mathrm{S}$ & $\mathrm{N}$ & $\mathrm{S}$ & 3 & E58 & $\mathrm{S}$ & $\mathrm{N}$ & $\mathrm{S}$ & $\mathrm{S}$ & 3 \\
\hline E22 & $\mathrm{S}$ & $\mathrm{S}$ & $\mathrm{S}$ & $\mathrm{N}$ & 3 & E59 & $\mathrm{S}$ & $\mathrm{S}$ & $\mathrm{S}$ & $\mathrm{N}$ & 3 \\
\hline E23 & $\mathrm{S}$ & $\mathrm{N}$ & $S$ & $\mathrm{~S}$ & 3 & E60 & $\mathrm{N}$ & $\mathrm{N}$ & $\mathrm{S}$ & $\mathrm{S}$ & 2 \\
\hline E24 & $\mathrm{N}$ & $\mathrm{N}$ & $S$ & $\mathrm{~S}$ & 2 & E61 & $\mathrm{N}$ & $\mathrm{N}$ & $\mathrm{S}$ & $\mathrm{S}$ & 2 \\
\hline E25 & $\mathrm{S}$ & $\mathrm{N}$ & $\mathrm{S}$ & $\mathrm{N}$ & 2 & E62 & $\mathrm{N}$ & $\mathrm{N}$ & $\mathrm{S}$ & $\mathrm{S}$ & 2 \\
\hline E26 & $\mathrm{S}$ & $\mathrm{N}$ & $\mathrm{S}$ & $\mathrm{S}$ & 3 & E63 & $\mathrm{N}$ & $\mathrm{N}$ & $\mathrm{S}$ & $\mathrm{S}$ & 2 \\
\hline E27 & $\mathrm{S}$ & $\mathrm{N}$ & $\mathrm{N}$ & $\mathrm{S}$ & 2 & E64 & $\mathrm{N}$ & $\mathrm{S}$ & $\mathrm{N}$ & $\mathrm{S}$ & 2 \\
\hline E28 & $\mathrm{S}$ & $\mathrm{N}$ & $\mathrm{N}$ & $\mathrm{S}$ & 2 & E65 & $\mathrm{N}$ & $\mathrm{N}$ & $\mathrm{S}$ & $\mathrm{S}$ & 2 \\
\hline E29 & $\mathrm{S}$ & $\mathrm{N}$ & $\mathrm{S}$ & $\mathrm{N}$ & 2 & E66 & $\mathrm{S}$ & $\mathrm{N}$ & $\mathrm{S}$ & $\mathrm{S}$ & 3 \\
\hline E30 & $\mathrm{S}$ & $\mathrm{N}$ & $\mathrm{S}$ & $\mathrm{S}$ & 3 & E67 & $\mathrm{S}$ & $\mathrm{N}$ & $\mathrm{P}$ & $\mathrm{P}$ & 2 \\
\hline E31 & $\mathrm{S}$ & $\mathrm{N}$ & $\mathrm{N}$ & $\mathrm{N}$ & 1 & E68 & $\mathrm{S}$ & $\mathrm{N}$ & $\mathrm{S}$ & $\mathrm{S}$ & 3 \\
\hline E32 & $\mathrm{S}$ & $\mathrm{N}$ & $\mathrm{S}$ & $\mathrm{S}$ & 3 & E69 & $\mathrm{S}$ & $\mathrm{N}$ & $\mathrm{S}$ & $\mathrm{N}$ & 2 \\
\hline E33 & $\mathrm{S}$ & $\mathrm{S}$ & $\mathrm{S}$ & $\mathrm{N}$ & 3 & E70 & $\mathrm{N}$ & $\mathrm{N}$ & $\mathrm{S}$ & $\mathrm{S}$ & 2 \\
\hline E34 & $\mathrm{S}$ & $\mathrm{N}$ & $\mathrm{P}$ & $\mathrm{S}$ & 2.5 & E71 & $\mathrm{N}$ & $\mathrm{S}$ & $\mathrm{S}$ & $\mathrm{S}$ & 3 \\
\hline E35 & $\mathrm{N}$ & $\mathrm{N}$ & $\mathrm{S}$ & $\mathrm{S}$ & 2 & E72 & $\mathrm{S}$ & $\mathrm{N}$ & $\mathrm{S}$ & $\mathrm{N}$ & 2 \\
\hline E36 & $\mathrm{S}$ & $\mathrm{S}$ & $\mathrm{P}$ & $\mathrm{S}$ & 3.5 & \multirow[t]{2}{*}{ E73 } & \multirow[t]{2}{*}{$\mathrm{S}$} & \multirow[t]{2}{*}{$\mathrm{S}$} & \multirow[t]{2}{*}{$\mathrm{N}$} & \multirow[t]{2}{*}{$\mathrm{S}$} & \multirow[t]{2}{*}{3} \\
\hline E37 & $\mathrm{S}$ & $\mathrm{S}$ & $\mathrm{N}$ & $\mathrm{S}$ & 3 & & & & & & \\
\hline
\end{tabular}

Tabela 4: Avaliação da qualidade de RSL. 


\begin{tabular}{|c|c|c|}
\hline Abordagem & Descrição & Estudos \\
\hline $\begin{array}{l}\text { Avaliação da } \\
\text { ferramenta }\end{array}$ & Avaliação de ferramenta de apoio a programação & 3 \\
\hline \multirow{19}{*}{$\begin{array}{l}\text { Ferramentas de } \\
\quad \text { Software }\end{array}$} & Ambiente de ensino a distância & 2 \\
\hline & Ambiente contendo um sistema de raciocínio baseado em casos & 1 \\
\hline & Ambiente online para programação de computadores & 3 \\
\hline & Ambiente inteligente para melhoria de assistência ao aluno & 2 \\
\hline & Ambiente de Apoio a Programação e Teste de Software & 1 \\
\hline & Ambiente para aprendizagem cooperativa & 3 \\
\hline & Ambientes Visuais de Programação 3D & 1 \\
\hline & Ferramenta de programação visual & 2 \\
\hline & Ferramenta com simulação e visualização de código, correção automática & 2 \\
\hline & Ferramenta para acompanhamento e análise de programas & 2 \\
\hline & Ferramenta de programação para deficientes visuais & 1 \\
\hline & Ferramenta de personalização e acompanhamento da resolução de exercícios & 1 \\
\hline & Ambiente lúdico de programação & 1 \\
\hline & Ferramenta para acompanhamento e análise de programas & 1 \\
\hline & Ferramenta de Personalização dos Exercícios auxiliada por um Agente Afetivo & 1 \\
\hline & $\begin{array}{l}\text { Ferramenta que usa técnicas de computação afetiva para detectar nível de frustração } \\
\text { dos alunos }\end{array}$ & 1 \\
\hline & $\begin{array}{l}\text { Ferramenta com gerenciamento de recursos didáticos e acompanhamento das práticas } \\
\text { laboratoriais }\end{array}$ & 3 \\
\hline & Ferramenta de autoria & 2 \\
\hline & Ferramenta para o acompanhamento de aprendizagem em Lógica Computacional & 1 \\
\hline \multirow[t]{6}{*}{ Metodologia } & Estratégias de aprendizagem motivacional e problemas em ensino de programação & 2 \\
\hline & Modelo para avaliação e acompanhamento da aprendizagem & 1 \\
\hline & Metodologia Baseada no Sistema Personalizado de Ensino & 1 \\
\hline & Uso de objetos de aprendizagem & 1 \\
\hline & Uso de DOJOs & 1 \\
\hline & Estratégia para melhoria na abstração de resolução de problemas & 3 \\
\hline \multirow[t]{2}{*}{ Jogos } & Uso de jogos & 14 \\
\hline & Avaliação de empírica de um jogo & 1 \\
\hline Robótica & Uso da robótica & 7 \\
\hline $\begin{array}{l}\text { Linguagem de } \\
\text { programação e } \\
\text { robótica }\end{array}$ & Linguagem de programação de microcontroladores, kit de robótica & 2 \\
\hline \multirow{2}{*}{$\begin{array}{l}\text { Linguagem de } \\
\text { programação }\end{array}$} & Linguagem de programação icônica & 1 \\
\hline & Linguagem de programação para estudantes surdos & 1 \\
\hline $\begin{array}{l}\text { Técnica de } \\
\text { avaliação }\end{array}$ & Estudo da taxonomia para elaboração de um instrumento de avaliação & 2 \\
\hline $\begin{array}{l}\text { Arquitetura } \\
\text { Pedagógica }\end{array}$ & Arquitetura pedagógica para o ensino de programação & 1 \\
\hline
\end{tabular}

Tabela 5: Abordagens propostas nos artigos.

\subsection{Quais os níveis de escolaridade focados por estas abordagens? Ou seja, as abor- dagens propostas estão sendo aplicadas nas escolas e/ou em universidades?}

Os artigos foram classificados de acordo com o nível de escolaridade para os quais as pesquisas se destinaram. Os níveis de escolaridade indicados nos estudos são apresentados no gráfico da Figura 1.
Verificou-se que aproximadamente $60 \%$ dos artigos abordaram programação no contexto da educação superior. Apenas $22 \%$ dos artigos apresentaram trabalhos no contexto do nível médio. Já $11 \%$ dos artigos destinam-se ao ensino fundamental e somente $3 \%$ das pesquisas destinam-se ao ensino técnico. Esta revisão ainda apresenta que $1 \%$ dos artigos se destina ao ensino técnico e superior e $3 \%$ ao ensino fundamental e médio (ver Figura 1). 


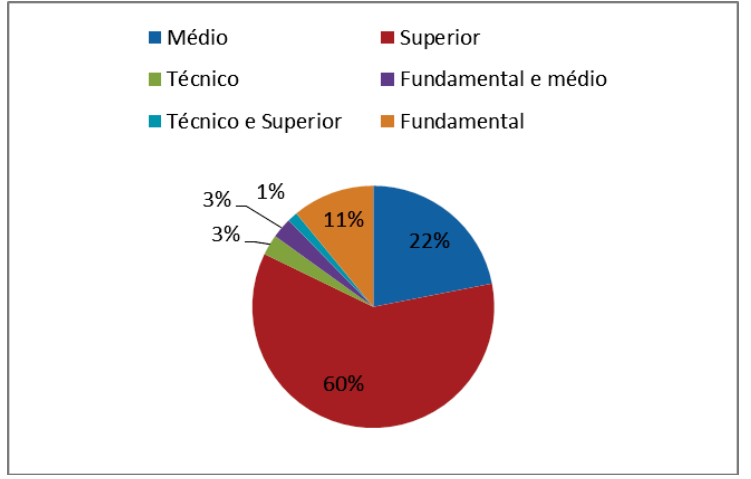

Figura 1: Níveis de escolaridades abordados nos estudos.

Nota-se ainda que, a maioria dos estudos está sendo aplicados nas universidades (aproximadamente 63\%). Apenas 34\% nas escolas e 3\% estão sendo aplicados tanto nas escolas, como nas universidades.

Desta forma, estes dados revelam a necessidade de mais pesquisas para alunos que estejam cursando o nível básico de ensino.

\subsection{Quais áreas de conhecimento estão sendo trabalhadas pelas abordagens propostas? Ou seja, existe relação entre estas abor- dagens e o conteúdo visto em sala de au- la?}

São várias as disciplinas que são trabalhadas nas pesquisas encontradas. No ensino superior, tem-se disciplinas de programação, como, introdução a programação (E1, E2, E5, E7, E12, E21), programação orientada a objetos (E9), lógica de programação (E10, E33 e E40), raciocínio lógico (E28 e E42) e estrutura de dados (E6 e 39).

Com relação às disciplinas relacionadas ao ensino fundamental e médio, somente 5 dos 73 trabalhos especificam as disciplinas. As disciplinas apresentadas por estes estudos são: matemática (E14, E21), matemática e física (E26), matemática, português e química (E53), geografia e matemática (E72) e português e matemática (E23). Nesses estudos, ocorre uma relação entre as abordagens apresentadas e o conteúdo visto nas escolas. Por exemplo, no estudo E72, à medida que o aluno aprende programação, ele também aprende conteúdos relacionados à matemática (pontos cardeais e plano cartesiano) e geografia (mapa de Santa Catarina).

\subsection{Quais abordagens pedagógicas estão sen- do utilizadas nos estudos?}

Esta questão tem como objetivo apontar as principais abordagens pedagógicas utilizadas nos estudos. As abordagens que foram citadas são apresentadas na Tabela 6 .

\begin{tabular}{|c|c|}
\hline $\begin{array}{l}\text { Abordagem } \\
\text { Pedagógica }\end{array}$ & Descrição \\
\hline $\begin{array}{l}\text { Aprendizagem } \\
\text { significativa }\end{array}$ & $\begin{array}{l}\text { Identifica o favorecimento da apren- } \\
\text { dizagem com a identificação das } \\
\text { relações entre os conceitos (E6 e } \\
\text { E36) [10]. }\end{array}$ \\
\hline $\begin{array}{l}\text { Pedagogia do } \\
\text { construtivismo }\end{array}$ & $\begin{array}{l}\text { Propõe que o aluno participe ativa- } \\
\text { mente do próprio aprendizado (E10, } \\
\text { E12, E20, E25 e E60) [12] [26]. }\end{array}$ \\
\hline $\begin{array}{l}\text { Taxionomia de } \\
\text { Bloom }\end{array}$ & $\begin{array}{l}\text { Tem a finalidade de classificar obje- } \\
\text { tivos da aprendizagem, de acordo } \\
\text { com um conjunto de três objetivos } \\
\text { educacionais: cognitivo, afetivo e } \\
\text { psicomotor (E24, E45, E47 e E58) } \\
\text { [4]. }\end{array}$ \\
\hline $\begin{array}{l}\text { Taxionomia } \\
\text { Revisada de } \\
\text { Bloom }\end{array}$ & $\begin{array}{l}\text { É um esquema de classificação pro- } \\
\text { veniente de alterações na taxonomia } \\
\text { de Bloom original. Embora tenha } \\
\text { mantido parte da estrutura original, a } \\
\text { taxonomia revisada é mais adequada } \\
\text { para suportar as novas formas de } \\
\text { aprendizagem (E71) [24]. }\end{array}$ \\
\hline $\begin{array}{l}\text { Abordagem } \\
\text { Construcionista }\end{array}$ & $\begin{array}{l}\text { Permite que o aprendiz construa o } \\
\text { seu próprio conhecimento por inter- } \\
\text { médio do computador (E30 e E59) } \\
\text { [27]. }\end{array}$ \\
\hline $\begin{array}{l}\text { Abordagem } \\
\text { Instrucionista }\end{array}$ & $\begin{array}{l}\text { Consiste na informatização dos mé- } \\
\text { todos de ensino tradicionais [28], ou } \\
\text { seja, em implementar um tutorial, } \\
\text { jogo, simulação, entre outros (E18). }\end{array}$ \\
\hline Ciclo de Kolb & $\begin{array}{l}\text { Este modelo se mostra adequado ao } \\
\text { ensino de programação, pela sua } \\
\text { abordagem experiencial e coerência } \\
\text { com as relações entre estilos de } \\
\text { aprendizagem e resolução de proble- } \\
\text { mas. Este ciclo envolve quatro etapas } \\
\text { de aprendizagem, são elas: sentir, } \\
\text { fazer, observar e pensar (E6) [9]. }\end{array}$ \\
\hline
\end{tabular}

Tabela 6: Abordagens pedagógicas apresentadas nos estudos.

Os demais estudos não apresentaram a utilização de nenhuma teoria pedagógica para auxiliar a metodologia de ensino de programação.

Além das abordagens pedagógicas que auxiliam o desenvolvimento dos estudos, alguns trabalhos utilizam também ferramentas, como apoio no desenvolvimento das pesquisas. Na Tabela 7 são apresentadas as ferramentas e os estudos encontrados nesta revisão. 


\begin{tabular}{|c|c|c|c|c|c|}
\hline Ferramenta & Estudos & Ferramenta & Estudos & Ferramenta & Estudos \\
\hline Flash CS5 & E3, E46, E55 & Alice & E32, E64 & $\begin{array}{c}\text { Squeak } \\
\text { Etoys }\end{array}$ & E12, E14 \\
\hline Scratch & $\begin{array}{c}\text { E8, E9, E15, E22, } \\
\text { E25, E27 }\end{array}$ & $\begin{array}{l}\text { Lego Mind- } \\
\text { storms NXT }\end{array}$ & $\begin{array}{l}\text { E26, E33, E34, E37, E42, } \\
\text { E44, E45, E68, E72, E73 }\end{array}$ & PyGame & E43, E53 \\
\hline Escracho & E59 & Visualg & E11 & Construct 2 & E21, E36 \\
\hline Takkou & E48 & Kodu & E31 & $\begin{array}{l}\text { Visual Stu- } \\
\text { dio }\end{array}$ & E5 \\
\hline iVprog & E29 & Game Maker & E57 & $\begin{array}{c}\text { BlueJ e } \\
\text { Greenfoot }\end{array}$ & E9 \\
\hline ProgTest & E30 & TutorICC & E58 & JavaTool & E47 \\
\hline Dosvox e Edivox & E56 & JColibri & E66 & Furbot & E50 \\
\hline
\end{tabular}

Tabela 7: Ferramentas utilizadas nos estudos.

\subsection{Quais habilidades e competências estão sendo exploradas nos alunos?}

Nos estudos foram encontradas três habilidades: cognitiva, social e emocional. Nas habilidades cognitivas foram apresentadas as seguintes características: resolver problemas; estabelecer conclusões lógicas e planejar e tomar decisões (E1, E3, E11, E13, E14, E16, E17, E18, E24, E25, E26, E29, E30, E31, E32, E33, E34, E36, E44, E45, E47, E48, E49, E55, E56, E57, E61, E67 e E71).

Já nas habilidades sociais, as características apresentadas foram: lidar com regras; cooperar e elaborar (E16, E21, E30, E37 e E47). As habilidades emocionais destacam: autoconfiança; autoestima e autoavaliação (E40).

Com relação às competências, somente dois estudos (E21 e E40) citaram competências, tais como: criatividade, estruturação do pensamento, responsabilidade, curiosidade, confiança e trabalho em equipe.

\subsection{Quais benefícios e limitações estão sendo relatados pelo uso das abordagens pro- postas?}

Através dos resultados obtidos pela RSL, observou-se que alguns benefícios e limitações foram relatados. Os benefícios relatados foram: o uso de estratégias como jogo e robótica motiva e melhora o ensino de programação, aumentando o poder de concentração dos alunos e a taxa de aprovação, bem como diminuição no número de desistências em disciplinas de programação; utilização de oficinas como forma lúdica e atrativa ao processo de ensino-aprendizagem de algoritmos; a inserção de novas ferramentas - como Scratch, Squeak Etoys e Kodu -, metodologias e técnicas, como forma de tornar o processo de ensino-aprendizagem mais dinâmico. Soluções lúdicas, como a utilização de linguagem de programação visual, contribuem para facilitar a aprendizagem dos alunos à medida que os auxiliam com resoluções de problemas.
No geral, as dificuldades enfrentadas pelos alunos não refletem algo incomum, pois estudantes de computação apresentam os mesmos problemas, principalmente, no início do curso: dificuldades de abstração e compreensão dos problemas; tratamento de erros; especificar e codificar uma solução. Detectou-se que o idioma inglês, comumente nativo das linguagens de programação, não é o problema chave no estudo de algoritmos. As dificuldades apontadas nas pesquisas pelos estudantes foram o entendimento do problema e a capacidade de raciocinar logicamente.

\subsection{As abordagens estão sendo destinadas à Educação Presencial e/ou à Distância?}

Esta questão tem como objetivo apontar em qual direção em relação ao ensino presencial ou à distância estão sendo destinadas as pesquisas.

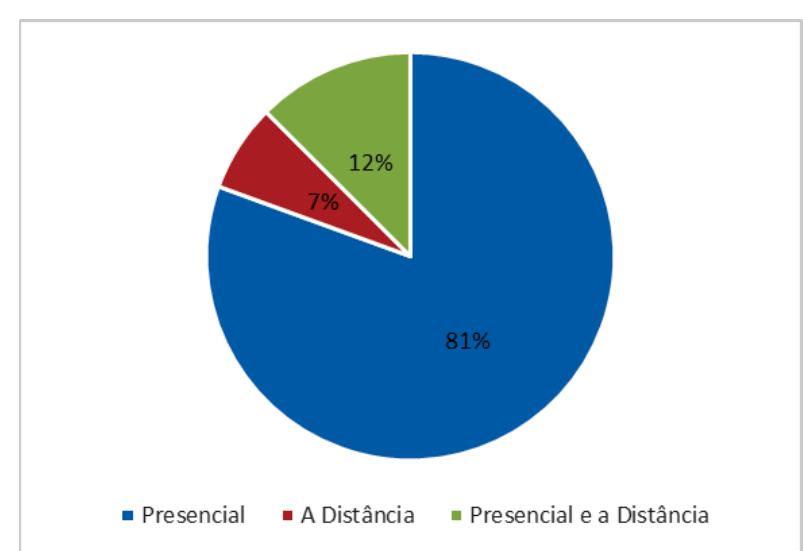

Figura 2. Porcentagem de aplicação dos estudos na educação presencial e a distância.

Nota-se ainda que a maioria dos estudos esteja sendo destinada ao ensino presencial, cerca de $81 \%$, como é ilustrado no gráfico da Figura 2. Apenas 12\% destinamse ao ensino à distância e $7 \%$ das abordagens podem ser utilizadas tanto na educação presencial como a distância. 


\subsection{Quais são as instituições de pesquisas envolvidas na área?}

Na Tabela 8 é apresentada a quantidade de artigos por instituições de pesquisa. Das 51 instituições com publicações, 14 delas tiveram mais de um trabalho publicado. Pode-se destacar a Universidade Federal da Paraíba (UFPB), que obtivem 14 artigos publicados, sendo 2 destes artigos em parceria, um com a UnB e o outro com uma faculdade paraibana, a IESP.

No gráfico da Figura 3 é ilustrada a distribuição geográfica das publicações por estado de origem. Dada a atuação da UFPB, o estado da Paraíba é responsável por $26 \%$ dos artigos publicados na área e analisado por esta revisão.

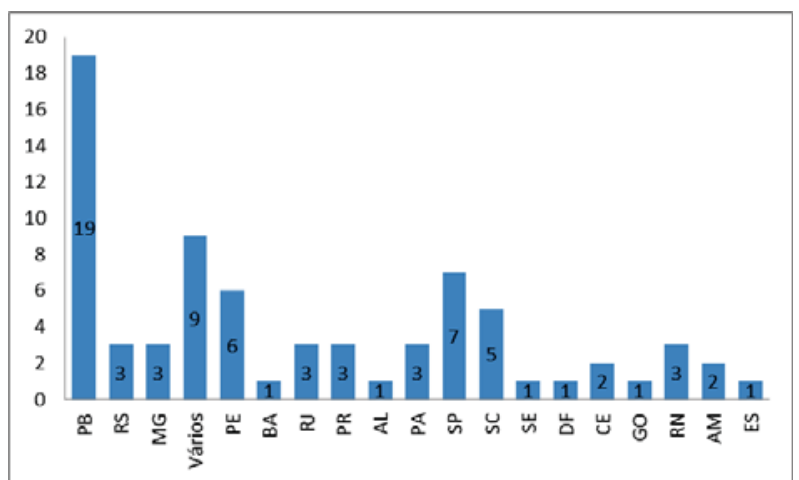

Figura 3. Distribuição geográfica dos artigos por estado.

Aproximadamente $45 \%$ das pesquisas apresentadas nos artigos foram realizadas por instituições localizadas na região Nordeste, $22 \%$ realizado na região Sul, $20 \%$ na região Sudeste, $9 \%$ na região Norte, e apenas $4 \%$ na região Centro-Oeste, como ilustra o gráfico da Figura 4. Um total de $15 \%$ dos artigos foi realizado em colaboração entre universidades. Três artigos foram realizados em colaboração instituições de pesquisa internacionais. Um artigo da UFRGS com a Universidade Nacional da Colômbia e dois da FURB com a Universiteit Van Amsterdam da Holanda.

A distribuição das instituições de pesquisa mostrou que a maior parte das publicações está localizada nas regiões Nordeste, Sul e Sudeste do Brasil. Na distribuição de artigos por evento, as instituições da região Nordeste são as que publicam mais nos eventos WEI e WIE, com $63 \%$ e $50 \%$, respectivamente. A região Sul, Sudeste, Norte e Centro-Oeste, têm respectivamente, $7 \%, 19 \%$, $7 \%$ e $4 \%$ das publicações no WEI e $17 \%, 17 \%, 8 \%$ e $8 \%$ no WIE. No SBIE a região Nordeste ainda predominar com $33 \%$ dos artigos, seguido de $30 \%$ da região Sul, $26 \%$ da região Sudeste, $7 \%$ da região Norte e $4 \%$ da região Centro-Oeste.

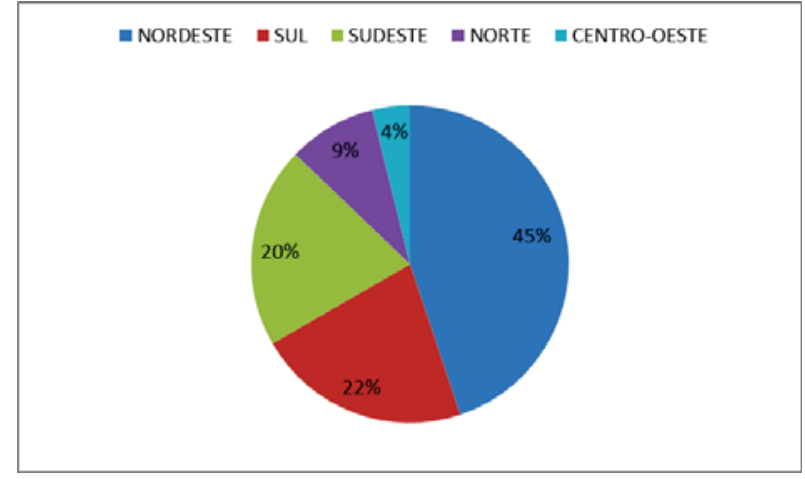

Figura 4. Distribuição geográfica de publicação por região.

Os dados ainda mostram que as pesquisas na área de ensino-aprendizagem de programação nos últimos cincos anos tem se mantido estável, como é ilustrado no gráfico da Figura 5. Na verdade, a quantidade de artigos publicados na área aumentou significativamente em 2013, tendo aproximadamente o dobro das publicações em relação aos anos anteriores.

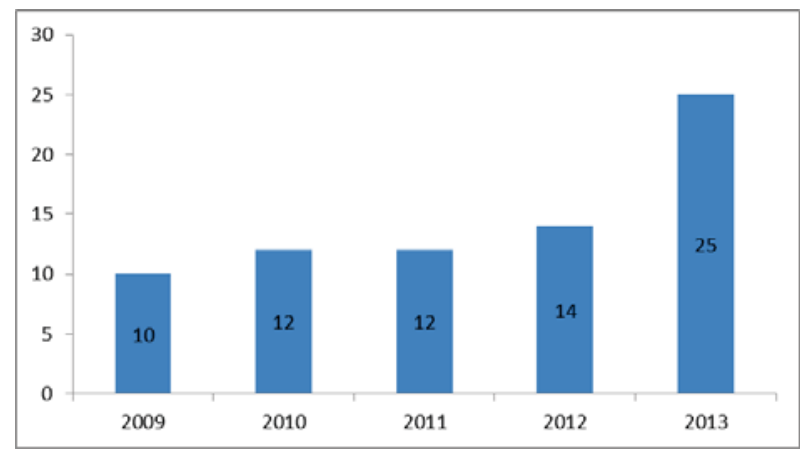

Figura 5. Distribuição anual dos estudos.

\subsection{Limitações da RSL}

Os procedimentos utilizados neste estudo se desviaram nos aspectos apresentados nas diretrizes de [8] em alguns pontos específicos:

- A pesquisa foi organizada como um processo de busca manual em um conjunto específico de periódicos e anais de congressos e não em um processo de busca automatizada, já que os eventos nacionais não são, em sua maioria, indexados por bases de artigos como as da ACM e IEEE;

- Um único pesquisador selecionou os estudos, baseado em seus títulos e resumos;

- Um único pesquisador tabulou os dados, apesar dos outros terem verificado a extração de dados, como sugerido por [5].

O primeiro ponto acima implica na possível ausência de estudos relevantes. Em particular, pode-se estar faltando artigos relevantes publicados em outras revistas e 
conferências nacionais. Essa ameaça foi controlada através da escolha dos principais eventos e revistas na área.

Com relação ao segundo ponto, como o número de artigos analisados durante a pré-seleção é elevado, há chances de que artigos relevantes tenham passado despercebidos na análise dos títulos, resumos e palavraschaves. Esta ameaça foi controlada fazendo-se uso de pesquisadores com experiência na área e tempo disponí- vel para fazer a seleção de forma cuidadosa.

O terceiro ponto indica que alguns dados coletados poderiam estar incorretos. O controle dessa ameaça foi realizado de forma cuidadosa por um pesquisador experiente em SRL e em análise desse tipo de dados. Além disso, esse terceiro ponto trata de uma quantidade reduzida de estudos primários, reduzindo o risco de erros.

\begin{tabular}{|c|c|c|c|c|c|}
\hline Instituição & Artigos & Instituição & Artigos & Instituição & Artigos \\
\hline UFPB & 12 & UFAL & 1 & UnB & 1 \\
\hline UFRGS & 3 & UEFS & 1 & CEETEPS & 1 \\
\hline UFRPE & 3 & UFAL & 1 & UFC & 1 \\
\hline UFPA & 3 & TECPAR/PUC-PR & 1 & UFG & 1 \\
\hline UFCG & 3 & UFPB/UnB & 1 & UTFPR & 1 \\
\hline UEPB & 2 & UEFS/UFGRS & 1 & UNISC & 1 \\
\hline UFF & 2 & UFPA/UFRA/UFES & 1 & FAA & 1 \\
\hline UFPE/IFPE & 2 & UFRJ & 1 & $\begin{array}{c}\text { FATEC- } \\
\text { ID/PUC- } \\
\text { CAMPINAS }\end{array}$ & 1 \\
\hline USP & 2 & PUC-PR & 1 & $\begin{array}{c}\text { UFRGS/SENA } \\
\text { C-PELOTAS }\end{array}$ & 1 \\
\hline $\begin{array}{c}\text { FURB, Universiteit } \\
\text { Van Amsterdam }\end{array}$ & 2 & ULBRA & 1 & UEM/CIT & 1 \\
\hline UNICAMP & 2 & UFRR/UEM & 1 & UDESC & 1 \\
\hline UFRN & 2 & UNIVALE & 1 & UNIFOR & 1 \\
\hline UNIVALI & 2 & UFS & 1 & $\begin{array}{l}\text { UERN- } \\
\text { UFERSA }\end{array}$ & 1 \\
\hline $\begin{array}{l}\text { UFRGS/Universidad } \\
\text { Nacional da Colôm- } \\
\text { bia }\end{array}$ & 1 & ITA & 1 & UFRA & 1 \\
\hline IFSULMINAS & 1 & \multirow[t]{2}{*}{ IESP/UFPB } & \multirow[t]{2}{*}{1} & \multirow[t]{2}{*}{ UFES } & \multirow[t]{2}{*}{1} \\
\hline UFPE/UPE & 1 & & & & \\
\hline
\end{tabular}

Tabela 8: Quantidade de artigos por instituição de pesquisa.

\section{Conclusões}

Nesta pesquisa, apresentamos os resultados de uma RSL sobre as abordagens para o ensino-aprendizagem de programação publicados nos últimos 5 anos no cenário nacional. Em particular no SBIE, WIE, WEI, SBGAMES, RENOTE e RBIE. A busca realizada nesta revisão resultou na pré-seleção de 105 artigos, dentre os quais 73 foram incluídos para a extração de dados.

A distribuição anual dos artigos mostra que de 2009 a 2012 as pesquisas nesta área tem se mostrado presentes de forma consistente. Já em 2013, 25 artigos foram publicados nessa temática nas revistas e congressos pesquisados, aproximadamente o dobro dos outros anos.

A distribuição geográfica das instituições de pesquisa mostrou que a maior parte das publicações está localizada nas regiões Nordeste, Sul e Sudeste do Brasil. Com destaque para a região Nordeste, correspondendo a $45 \%$ das pesquisas nesta temática. Na região Nordeste, destaca-se o enfoque das pesquisas no estado da Paraíba, correspondendo a $26 \%$ das publicações e a UFPB, como a universidade com mais artigos publicados, perfazendo um total 
de $16 \%$ dos estudos. Além disso, 15\% das pesquisas foram realizadas colaborativamente, contando com universidades localizadas em diversos estados brasileiros. Já três artigos contaram com a colaboração de universidades internacionais, sendo um artigo da UFRGS em parceria com a Universidad Nacional de Colômbia e dois da FURB, em colaboração com a universidade holandesa Universiteit Van Amsterdam.

É observada claramente uma maior publicação de artigos no contexto da educação superior. Estes dados mostram a necessidade de mais pesquisas que abordem o ensino de programação nos níveis fundamental, médio e técnico. Esses mesmos dados mostram também a necessidade de pesquisas para o contexto da Educação à Distância, visto que poucos são os trabalhos focam nessa abordagem de ensino.

Sobre os tipos de abordagens utilizadas, destaca-se uma preferência por se desenvolver ferramentas para o processo de ensino-aprendizagem de programação. Podese analisar que $11 \%$ dos estudos foram realizados com a utilização da robótica. O alto esforço para executar e gerenciar estudos com robótica, devido aos custos para comprar de robôs e kit de robótica, às vezes impossibilita a sua utilização em escolas do ensino fundamental e médio. A proposta de se utilizar jogos digitais para o ensino-aprendizagem de programação também é destacada em outros estudos. Em sua maioria, os jogos digitais de ensino de programação estão voltados para melhorar o rendimento dos alunos em disciplinas introdutórias de programação.

Acerca das abordagens pedagógicas utilizadas, temos, a Aprendizagem Significativa, Taxionomia de Bloom, Taxionomia Revisada de Bloom, Pedagógica do Construtivismo, Ciclo de Kolb e as abordagens Construcionista e Instrucionista.

Através dos resultados apresentados, este artigo contribuiu com uma visão geral do panorama nacional capaz de servir de ponto de partida para várias outras pesquisas, incentivando principalmente pesquisas nos níveis médio, fundamental e técnico, e também no contexto do Ensino à Distância, e na aplicação e avaliação destes trabalhos nas escolas.

\section{Agradecimentos}

Os autores agradecem a CAPES e ao CNPq pelo suporte parcial a esta pesquisa.

\section{Referências}

[1] A-F. Lai; S.-H G. The flow and self-efficacy of sixth grade students under Scratch programming learning. In: International Conference Electrical and Control Engineering, pp.6915,6919, 2011.

[2] V. C. O. Aureliano; P. C. A. R. Tedesco. Ensinoaprendizagem de Programação para Iniciantes: uma Revisão Sistemática da Literatura focada no SBIE e WIE. In: XXII Simpósio Brasileiro de Informática na Educação, Rio de Janeiro, 2012.

[3] A. C. Barros; J. Wainer; K. Claudio; L. R. R. Ferreira; T. Dwyer. Uso de computadores no ensino fundamental e médio e seus resultados empíricos: uma revisão sistemática da literatura. In: Revista Brasileira de Informática na Educação, v.16, n.1, 2008.

[4] B. S. Bloom; M. D. Engelhart; E. J. Furst; W. H. Hill; D. R. Krathwohl. Taxonomia de objetivos educacionais - domínio cognitivo. Globo: Porto Alegre - RS. 1977.

[5] O. P. Brereton, B. A. Kitchenham, D. Turner Budgen, M. Khalil. Lessons from applying the systematic literature review process within the software engineering domain. In: Journal of Systems and Software, v.80, n.4, p.571-583, 2007.

[6] E. A. Jesus; A. L. A, Raabe. Interpretações da Taxonomia de Bloom no Contexto da Programação Introdutória. In: XX Simpósio Brasileiro de Informática na Educação, 2009.

[7] N. M. Diah; M. Ismail; S. Ahmad; S Abdullah. Jawi on Mobile devices with Jawi wordsearch game application. In: International Conference Science and Social Research, pp.326-329, 2010.

[8] B, Kitchenham. Guidelines for performing Systematic Literature Reviews in Software Engineering, version 2.3. Technical Report EBSE. Software Engineering Group. School of Computer Science and Mathematics Keele University. 2007.

[9] D. A. Kolb; R. E. Boyatzis; C. Mainemelis. Experiential Learning Theory: Previous Research and New Directions. In: Perspectives on cognitive, learning, and thinking styles. Sternberg \& Zhang (Eds.). NJ: Lawrence Erlbaum. 2000.

[10] D. P. Ausubel. Psicología educativa: un punto de vista cognoscitivo. Trad. Roberto Helier Domínguez. Trillas: México, 1976.

[11] C. Paes; J. Aguiar; M. Romão; A. Mendonça. Estratégias de Tutoria em um Curso à Distância de Programação para Alunos do Ensino Médio. In: Workshop de Informática na Escola, 2010, pp.1445-1448. 
[12] J. Piaget. Epistemologia genética. Tradução de Álvaro Cabral. 3. ed. São Paulo: Martins Fontes, 2007.

[13] D. Piva Júnior; R. L. Freitas. Estratégias para melhorar os processos de abstração na disciplina de Algoritmos. In: XX Simpósio Brasileiro de Informática na Educação, 2010.

[14] C. G. R. Ramirez; J. B. Almonte; R. R. Tugade; R. O. Atienza. Implementation of a digital gamebased learning environment for elementary Education. In: 2nd International Conference Education Technology and Computer, v.4, pp.22-24, 2010.

[15] A. V. M. Souto; M. Duduchi. Um processo de avaliação baseado em ferramenta computadorizada para o apoio ao ensino de programação de computadores. In: Workshop de Educação em Computação, 2009.

[16] S. Yamamoto; T. Tetsui; M. Naganuma; R. Kimura. Trial of Using Robotic Pet as Human Interface of Multimedia Education System for Pre-school Aged Child in Kindergarten. International Joint Conference SICE-ICASE, pp.33983403, 2006.

[17] J. Pereira Júnior; C. E. Rapkiewicz; C. Delgado; J. A. M. Xexeo. Ensino de Algoritmos e Programação: Uma Experiência no Nível Médio. XIII Workshop de Educação em Computação. São Leopoldo, RS. 2005.

[18] R. França; W. Silva; H. Amaral. Ensino de Ciência da Computação na Educação Básica: Experiências, Desafios e Possibilidades. In: $X X$ Workshop de Educação em Computação. Curitiba, PR. 2012.

[19] P. D. Scaico, A. A. Lima, J. B. B. Silva, S. Azevedo, L. F. Paiva, E. H. Raposo, Y. Alencar, J. P. Mendes, A. Scaico. In: Ensino de Programação no Ensino Médio: Uma Abordagem Orientada ao Design com a linguagem Scratch. In: Revista Brasileira de Informática na Educação, v.21, n.2, p.92-103, 2013.

[20] M. R. G. Barbosa; F. A. Silva; V. M. A. Oliveira; V. D. Feltrim; L. G. B. Mirisola; P. C. Gonçalves; J. J. G. Ramos; L. T. Alves. Implementação de Compilador e Ambiente de Programação Icônica para a Linguagem Logo em um Ambiente de Robótica Pedagógica de Baixo Custo. In: XX Simpósio Brasileiro de Informática na Educação, 2009.

[21] C. J. Santos; Franco, M; Corsini, H. C. C. AL-
Gbr: Uma Nova Ferramenta para Apoio ao Ensino/Aprendizagem de Lógica Computacional por Meio da Construção e Testes de Algoritmos. In: XXI Workshop Sobre Educação em Computação, 2013.

[22] M. H. Pietruchinski; J. Coelho Neto; A. Malucelli; S. Reinehr. Os jogos educativos no contexto do SBIE: uma revisão sistemática de Literatura. In: XXII Simpósio Brasileiro de Informática na Educação, p.476-485, 2011.

[23] T. J. Medeiros, T. R. Silva, E. H. S. Aranha. Ensino de programação utilizando jogos digitais: uma revisão sistemática da literatura. In: Revista Novas Tecnologias na Educação, v.11, n.3, 2013.

[24] E. A. Jesus, A. L. A. Raabe. Interpretações da Taxonomia de Bloom no Contexto da Programação Introdutória. In: XX Simpósio Brasileiro de Informática na Educação, Florianópolis, Brasil, 2009.

[25] T. R. Silva, T. J. Medeiros, E. H. S. Aranha. Jogos Digitais para Ensino e Aprendizagem de Programação: uma Revisão Sistemática da Literatura. In: XXV Simpósio Brasileiro de Informática na Educação, Dourados, Brasil, 2014.

[26] J. Bruner. A cultura da educação. Porto Alegre: Artmed, 2001.

[27] S. Papert. Constructionism: A new opportunity for elementary science education. A proposal to the National Science Foundation, Massachusetts Institute of Technology, Media Laboratory, Epistemology and Learning Group, Cambridge, Massachusetts. 1986.

[28] J. A. Valente. Informática na educação: instrucionismo x construcionismo. 1997. Disponível em:

$<$ http://www.educacaopublica.rj.gov.br/bibliotec a/tecnologia/0003.html $>$. Acessado em: $18 \mathrm{de}$ novembro de 2014 .

[29] P. Olson. Why Estonia Has Started Teaching Its First-Graders To Code. Disponível em: $<$ http://www.forbes.com/sites/parmyolson/2012/ 09/06/why-estonia-has-started-teaching-its-firstgraders-to-code/ $>$. Acessado em: 05 de novembro de 2014.

[30] A. Tucker. Model Curriculum for K-12 Computer Science. Final Report of the ACM K-12 Task Force Curriculum Committee. Association for Computing Machinery (ACM) 2004. 


\section{Apêndice A - Lista de referências dos artigos selecionados (em ordem crono- lógica)}

(E1) M. Maia; P. Scaico; F. Sousa; H. Peixoto. Uso de Estratégias de Aprendizagem e Motivacionais de Alunos em Disciplinas de Programação: Um Estudo de Caso na Licenciatura em Computação. In: XXI Workshop sobre Educação em Computação, Maceió, Brasil, 2013.

(E2) T. Costa; F. Bublitz. Análise dos Principais Problemas que Afetam Alunos de Programação: uma investigação empírica no Estado da Paraíba. . In: XXI Workshop sobre Educação em Computação, Maceió, Brasil, 2013.

(E3) C. Kahwage; E. L. França; R. Nunes. Jogo Baralho das Variáveis In: XXI Workshop sobre Educação em Computação, Maceió, Brasil, 2013.

(E4) R. S. Rodrigues; L. A. M. Morais; S. Dantas; J. G. Lopes Filho; C. Isidro; P. Suárez. Ensino de Algoritmos e Linguagem de Programação no Nível Médio: Um Relato de Experiência. In: XXI Workshop sobre Educação em Computação, Maceió, Brasil, 2013.

(E5) C. J. Santos; M. Franco; H. C. C. Corsini. ALGbr: Uma Nova Ferramenta para Apoio ao Ensino/Aprendizagem de Lógica Computacional por Meio da Construção e Testes de Algoritmos. In: XXI Workshop sobre Educação em Computação, Maceió, Brasil, 2013.

(E6) A. Santos. Uma Abordagem Integrativa para o Ensino de Algoritmos a Distância In: XXI Workshop sobre Educação em Computação, Maceió, Brasil, 2013.

(E7) P. Scaico; V. Dantas; F. Cunha; A. Farias; M. Henrique; F. M. Oliveira. ATIVA: Atividades Virtuais de Apoio Aplicadas ao Ensino de Programação. In: XXI Workshop sobre Educação em Computação, Maceió, Brasil, 2013.

(E8) V. Aureliano; P. Tedesco. Utilizando o Scratch para Apoiar o Processo de Ensino-Aprendizagem de Programação para Iniciantes na EaD. In: XXI Workshop sobre Educação em Computação, Maceió, Brasil, 2013

(E9) R. Bittencourt. Aprendizagem de Programação Através de Ambientes Lúdicos em um Curso de Engenharia de Computação: Uma Primeira Incursão. In: XXI Workshop sobre Educação em Computação, Maceió, Brasil, 2013.

(E10) O. Tavares; C. Menezes; R. A. Nevado; L. B. Costa. Arquiteturas Pedagógicas com Recursos Tecnológicos para Facilitarem a Aprendizagem de Programação. In: XXI Workshop sobre Educação em Computação, Maceió, Brasil, 2013.

(E11) M. B. Souza; J. L. G. Moreira; F. L. Lobo. Uma Abordagem Metodológica voltada para o Ensino-Aprendizagem de Algoritmos. In: Revista Novas Tecnologias na Educação, V.11, N.1, 2013.

(E12) B. F. Sperb; P. B. Schäfer; L. C. Fagundes. Squeak Etoys na modalidade 1 para 1: programação e autoria multimídia no desenvolvimento da conceituação. In: Revista Brasileira de Informática na Educação, V.21, N.1, 2013.
(E13) M. Souza, E. V. Jaeger, B. M. S. Cardoso. Ensino de algoritmos apoiados pelo uso de jogos digitais educativos. In: Novas Tecnologias na Educação, v.11, n.3, 2013.

(E14) A. D. Morais, L. C. Fagundes, M. V. A. Basso. Reflexões sobre o raciocínio lógico ao aprender a programar no Squeak Etoys. In: Revista Novas Tecnologias na Educação, v.11, n.3, 2013.

(E15) P. D. Scaico; A. A. Lima; J. B. B. Silva; S. Azevedo; L. F. Paiva; E. H. Raposo; Y. Alencar; J. P. Mendes; A. Scaico. Ensino de Programação no Ensino Médio: Uma Abordagem Orientada ao Design com a linguagem Scratch.. In: Revista Brasileira de Informática na Educação, V.21, N.2, 2013.

(E16) H. P. Pontes. Desenvolvimento de Jogos no Processo de Aprendizado em Algoritmos e Programação de Computadores. In: XII Simpósio Brasileiro de Jogos e Entretenimento Digital, São Paulo, Brasil, 2013.

(E17) T. S. C. da Silva; J. C. B. Melo. Cidade dos Bits: Um game para auxiliar no Aprendizado dos Fundamentos da Ciência da Computação a Nível Médio. In: XXIV Simpósio Brasileiro de Informática na Educação, Campinas, Brasil, 2013.

(E18) V. F. Dantas; E. R. de Macedo; J. R. B. Andrade; D. R. A. Coutinho; A. F. Cavalcante; T. G. Vasconcelos; M. E. S. Pereira. Combinando desafios e aventura em um jogo para apoiar a aprendizagem de programação em vários níveis cognitivos. In: XXIV Simpósio Brasileiro de Informática na Educação, Campinas, Brasil, 2013.

(E19) M. Gaudencio; L. F. Wanderley; F. W. Lemos; E. C. Araújo; J. C. A. Figueiredo; D. D. S. Guerrero. Eu Sei o que Vocês Fizeram (Agora e) na Aula Passada: o TSTView no Acompanhamento de Exercícios de Programação. In: XXIV Simpósio Brasileiro de Informática na Educação, Campinas, Brasil, 2013.

(E20) J. O. M. Chaves; A. F. Castro; R. W. Lima; M. V. A. Lima; K. H. A. Ferreira. Integrando Moodle e Juízes Online no Apoio a Atividades de Programação. In: XXIV Simpósio Brasileiro de Informática na Educação, Campinas, Brasil, 2013.

(E21) L. A. Melo; T. K. L. Costa; A. C. D. Batista. Pense bem: proposta e desenvolvimento de jogo digital para ensino de computação na educação básica. In: XXIV Simpósio Brasileiro de Informática na Educação, Campinas, Brasil, 2013.

(E22) A. L. S. O. Araújo; P. D. Scaico; L. F. Paiva; H. M. Rabêlo; L. L. Santos; F. I. R. Pessoa; J. M. Targino; L. S. Costa. Aplicação da Taxonomia de Bloom no ensino de programação com Scratch. In: XIX Workshop de Informática na Escola, Campinas, Brasil, 2013.

(E23) D. F. Sousa. Desenvolvendo a Lógica e Algoritmos no Ensino Médio. In: XIX Workshop de Informática na Escola, Campinas, Brasil, 2013

(E24) C. Torezani; L. B. C. Chagas; O. L. Tavares. NewProg - um ambiente online para crianças aprenderem programação de computadores. In: XIX Workshop de Informática na Escola, Campinas, Brasil, 2013.

(E25) R. S. França; H. J. C. Amaral. Proposta Metodológica de Ensino e Avaliação para o Desenvolvimento do Pensamento Computacional com 
o Uso do Scratch. In: XIX Workshop de Informática na Escola, Campinas, Brasil, 2013.

(E26) V. N. Silva; M. N. Nascimento. Investigação da melhoria do aprendizado de alunos do ensino médio da rede pública de ensino através do uso de programação, robótica e jogos digitais. In: Simpósio Brasileiro de Jogos e Entretenimento Digital, p.176-179, 2012

(E27) V. C. O. Aureliano; P. C. A. R. Tedesco. Avaliando o uso do Scratch como abordagem alternativa para o processo de ensinoaprendizagem de programação. In: XX Workshop sobre Educação em Computação, Curitiba, Brasil, 2012.

(E28) P. D. Scaico; D. Lopes; M. A. Azevedo; J. C. Silva; S. V. Mendes Neto; E. S. F. Falcão. Implementação de um Jogo Sério para o Ensino de Programação para Alunos do Ensino Médio Baseado em m-learning. In: XX Workshop sobre Educação em Computação, Curitiba, Brasil, 2012 .

(E29) R. S. R; L. O. Brandão; A. A. F. Brandão. Uma visão do cenário Nacional do Ensino de Algoritmos e Programação: uma proposta baseada no Paradigma de Programação Visual. In: XXIII Simpósio Brasileiro de Informática na Educação, Rio de Janeiro, Brasil, 2012.

(E30) D. M. Souza; J. C. Maldonado; E. F. Barbosa. Aspectos de Desenvolvimento e Evolução de um Ambiente de Apoio ao Ensino de Programação e Teste de Software. In: XXIII Simpósio Brasileiro de Informática na Educação, Rio de Janeiro, Brasil, 2012.

(E31) P. R. A. Souza; L. R. Dias. Kodu Game Labs: Estimulando o Raciocínio Lógico através de Jogos. . In: XXIII Simpósio Brasileiro de Informática na Educação, Rio de Janeiro, Brasil, 2012.

(E32) J. Valaski; E. C. Paraiso. Limitações da Utilização do Alice no Ensino de Programação para Alunos de Graduação. In: XXIII Simpósio Brasileiro de Informática na Educação, Rio de Janeiro, Brasil, 2012.

(E33) R. V. Friedrich; D. S. Santos; R. S. Keller; M. D. Puntel; D. Biasoli. Proposta Metodológica para a Inserção ao Ensino de Lógica de Programação com Logo e Lego Mindstorms. . In: XXIII Simpósio Brasileiro de Informática na Educação, Rio de Janeiro, Brasil, 2012.

(E34) P. Scaico; A. C. Duarte; G. M. T. Alves; M. Maia; F. M. Oliveira; S. V. M. Neto, M. R. X. Laurentino; A. Scaico; V. F. Dantas. Relato de um modelo de tutoria para programação baseado em experiências com ingressantes de um curso de Licenciatura em Computação. In: XX Workshop sobre Educação em Computação, Curitiba, Brasil, 2012.

(E35) P. D. Scaico; A. A. Lima; J. B. B. Silva; S. Azevedo; L. F. Paiva; E. H. S. Raposo; Y. Alencar; J. P. Mendes. Programação no Ensino Médio: Uma Abordagem de Ensino Orientado ao Design com Scratch.. In: XVIII Workshop de Informática na Escola, Rio de Janeiro, 2012.

(E36) P. Scaico; D. L. Marques; L. A. Melo; M. A. Azevedo; S. V. Mendes Neto; A. Oliveira; J. Alves Júnior; M. Labanca; A. Scaico. Um jogo para o ensino de programação em Python baseado na taxonomia de Bloom. In: XX Workshop sobre Educação em Computação, Curitiba, Brasil, 2012.

(E37) M. E. J. K. Cruz; R. Frozza; S. G. Marques; R. R. Viana; R. F. Molz; T. Rebelatto. Simulador para Introdução da Programação para
Crianças e Análise da Aprendizagem com apoio da Neurociência. In: Revista Novas Tecnologias na Educação, V.10, N.3, 2012.

(E38) T. Costa; A. Batista; M. Maia; L. Almeida; A. Farias. Trabalhando Fundamentos de Computação no Nível Fundamental: experiência de licenciandos em Computação da Universidade Federal da Paraíba. In: XX Workshop sobre Educação em Computação, Curitiba, Brasil, 2012.

(E39) D. H. Carmo; V. Braganholo. Um estudo sobre o uso didático de DOJOs de programação. In: XX Workshop sobre Educação em Computação, Curitiba, Brasil, 2012

(E40) E. F. Iepsen; M. Bercht; E. Reategui. Detecção e Tratamento do Estado Afetivo Frustração do Aluno na Disciplina de Algoritmos. In: XXII Simpósio Brasileiro de Informática na Educação, Aracaju, Brasil, 2011

(E41) A. B. França; J. M. Soares; D. G. Gomes; G. C. Barroso. Um sistema orientado a serviços para suporte a atividades de laboratório em disciplinas de técnicas de programação com integração ao ambiente Moodle. In: Revista Novas Tecnologias na Educação, V.9, N.1, 2011.

(E42) P. C. Ribeiro; C. B. Martins; F. C. Bernardini. A Robótica como Ferramenta de Apoio ao Ensino de Disciplinas de Programação em Cursos de Computação e Engenharia.. In: XVII Workshop de Informática na Escola, Aracaju, Brasil, 2011.

(E43) D. L. Marques; L. F. S. Costa; M. A. A. Silva; A. D. D. S. Rebouças. Atraindo Alunos do Ensino Médio para a Computação: Uma Experiência Prática de Introdução a Programação utilizando Jogos e Python. In: XVII Workshop de Informática na Escola, Aracaju, Brasil, 2011.

(E44) R. F. Soares; M. A. F. Borges. Robótica: aprendizado em informática de forma lúdica. In: XIX Workshop sobre Educação em Computação, Natal, Brasil, 2011.

(E45) T. S. C. Silva; A. S. C. Silva; J. C. B. Melo. Ensino de Algoritmos a Nível Médio Utilizando Música e Robótica: Uma Abordagem Lúdica. In: XIX Workshop sobre Educação em Computação, Natal, Brasil, 2011.

(E46) R. F. Azevedo; B. C. Paula. Proposta de metodologia de aprendizado da programação de computadores através da recontextualização de jogos sérios no estilo Game \& Watch. In: Simpósio Brasileiro de Jogos e Entretenimento Digital, Salvador, Brasil, 2011.

(E47) S. Sirotheau; S. R. Brito; A. S. Silva; M. K. Eliasquevici; E. L. Favero; O. L. Tavares. Aprendizagem de iniciantes em algoritmos e programação: foco nas competências de autoavaliação. In: XXII Simpósio Brasileiro de Informática na Educação, Aracaju, Brasil, 2011.

(E48) L. S. Barbosa; T. C. B. Fernandes; A. M. C. Campos. Takkou: Uma Ferramenta Proposta ao Ensino de Algoritmos. In: XIX Workshop sobre Educação em Computação, Natal, Brasil, 2011.

(E49) J. C. S. Santos; A. R. L. Ribeiro. JOnline: proposta preliminar de um juiz online didático para o ensino de programação. In: XXII Simpósio Brasileiro de Informática na Educação, Aracaju, Brasil, 2011.

(E50) G. C. Silva; R. Ré; A. Kawamoto; A. Schwerz. Uma Experiência na Aplicação de Práticas de Apoio no Ensino-Aprendizado de Algoritmos. In: XVII Workshop de Informática na Escola, Aracaju, Brasil, 
2011

(E51) R. E. S. Santos; C. V. C. Magalhães; J. S. Correia Neto; S. S. L. Paiva Júnior. PROGLIB: Uma Linguagem de Programação Baseada na Escrita de LIBRAS. In: XVII Workshop de Informática na Escola, Aracaju, Brasil, 2011.

(E52) J. Moreno. Uso de juegos digitales educativos como herramienta de soporte para el aprendizaje de algoritmos. In: Revista Novas Tecnologias na Educação, V.8, N.3, 2010.

(E53) A. D. D. S. Rebouças; D. L. Marques; L. F. S. Costa; M. A. A. Silva. Aprendendo a Ensinar Programação Combinando Jogos e Python. In: XXI Simpósio Brasileiro de Informática na Educação, João Pessoa, Brasil, 2010.

(E54) P. S. Rocha; B. Ferreira; D. Monteiro; D. S. C. Nunes; H. C. N. Góes. Ensino e Aprendizagem de Programação: Análise da Aplicação de Proposta Metodológica Baseada no Sistema Personalizado de Ensino. In: Revista Novas Tecnologias na Educação, V.8, N.3, 2010.

(E55) I. B. Saraiva; C. Mendes Netto. Monitor: um conjunto de objetos de aprendizagem para apoio ao ensino de programação de computadores. In: XVIII Workshop sobre Educação em Computação, Belo Horizonte, Brasil, 2010.

(E56) H. M. Silveira; L. C. Martini. MATVOX: um aplicativo para deficientes visuais que proporciona a implementação de algoritmos e cálculos matemáticos em um editor de texto. In: XXI Simpósio Brasileiro de Informática na Educação, João Pessoa, Brasil, 2010.

(E57) C. G. Sales; V. F. Dantas. ProGame: um jogo para o ensino de algoritmos e programação. In: XXI Simpósio Brasileiro de Informática na Educação, João Pessoa, Brasil, 2010.

(E58) H. L. Píccolo; V. F. Sena; K. B. Nogueira; M. O. Silva; Y. A. N. Maia. Ambiente Interativo e Adaptável para ensino de Programação. In: XXI Simpósio Brasileiro de Informática na Educação, João Pessoa, Brasil, 2010.

(E59) E. A. Jesus; A. L. A. Raabe. Avaliação Empírica da Utilização de um Jogo para Auxiliar a Aprendizagem de Programação. In: XXI Simpósio Brasileiro de Informática na Educação, João Pessoa, Brasil, 2010.

(E60) C. Paes; J. Aguiar; M. Romão; A. Mendonça. Estratégias de Tutoria em um Curso à Distância de Programação para Alunos do Ensino Médio. In: XVI Workshop de Informática na Escola, João Pessoa, Brasil, 2010.

(E61) D. Piva Júnior; R. L. Freitas. Estratégias para melhorar os processos de abstração na disciplina de Algoritmos. In: XXI Simpósio Brasileiro de Informática na Educação, João Pessoa, Brasil, 2010.

(E62) R. Pitta; S. Thomaz; A. Aglaé; S. Azevedo; A. Burlamaqui; L. M. Gonçalves. RoboEduc: Um Software para Programação em Níveis. In: XVI Workshop de Informática na Escola, João Pessoa, Brasil, 2010.

(E63) E. F. Iepsen; M. Bercht; E. Reategui. Persona-Algo: Personalização dos Exercícios de Algoritmos auxiliados por um Agente Afetivo. In: XVI Workshop de Informática na Escola, João Pessoa, Brasil, 2010.

(E64) H. W. A. S. Gondim; A. P. Ambrósio; F. M. Costa. Uma Experi- ência no Ensino de Algoritmos utilizando Ambientes Visuais de Programação 3D. In: XVII Workshop sobre Educação em Computação, Bento Gonçalves, Brasil, 2009.

(E65) M. P. Mota; S. R. Brito; M. P. Moreira; E. L. Favero. Ambiente Integrado à Plataforma Moodle para Apoio ao Desenvolvimento das Habilidades Iniciais de Programação. In: XX Simpósio Brasileiro de Informática na Educação, Florianópolis, Brasil, 2009.

(E66) G. P. Santos Júnior; J. M. Fechine; E. B. Costa. Analogus: Um Ambiente para Auxílio ao Ensino de Programação Orientado pelo Raciocínio por Analogia. In: XVII Workshop sobre Educação em Computação, Bento Gonçalves, Brasil, 2009.

(E67) A. Vahldick; F. B. V. Benitti; D. L. Urban; M. L. Krueger; A. Halma. O uso do Lego Mindstorms no apoio ao Ensino de Programação de Computadores. In: XVII Workshop sobre Educação em Computação, Bento Gonçalves, Brasil, 2009.

(E68) F. Sievers Júnior; J. S. E. Germano; F. Almeida. Kit Programa Fácil - um kit educacional para subsidiar a programação de microcomputadores em sala de aula. In: XX Simpósio Brasileiro de Informática na Educação, Florianópolis, Brasil, 2009.

(E69) A. V. M. Souto; M. Duduchi. Um processo de avaliação baseado em ferramenta computadorizada para o apoio ao ensino de programação de computadores. In: XVII Workshop sobre Educação em Computação, Bento Gonçalves, Brasil, 2009.

(E70) O. Hinterholz Júnior. Tepequém - uma nova Ferramenta para o Ensino de Algoritmos nos Cursos Superiores em Computação. In: XVII Workshop sobre Educação em Computação, Bento Gonçalves, Brasil, 2009.

(E71) E. A. Jesus; A. L. A. Raabe. Interpretações da Taxonomia de Bloom no Contexto da Programação Introdutória. In: XX Simpósio Brasileiro de Informática na Educação, Florianópolis, Brasil, 2009.

(E72) F. B. V. Benitti; A. Vahldick; D. L. Urban; M. L. Krueger; A. Halma. Experimentação com Robótica Educativa no Ensino Médio: ambiente, atividades e resultados. In: XV Workshop de Informática na Escola, Florianópolis, Brasil, 2009.

(E73) M. R. G. Barbosa; F. A. Silva; V. M. A. Oliveira; V. D. Feltrim; L. G. B. Mirisola; P. C. Gonçalves; J. J. G. Ramos; L. T. Alves. Implementação de Compilador e Ambiente de Programação Icônica para a Linguagem Logo em um Ambiente de Robótica Pedagógica de Baixo Custo. In: XX Simpósio Brasileiro de Informática na Educação, Florianópolis, Brasil, 2009. 